\title{
Enzyme evolution at home
}

Green Chem. http://doi.org/cxff (2018)

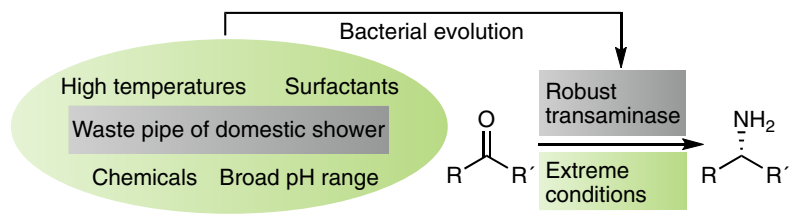

Chiral amines are essential components of many drugs and other valuable compounds. Biocatalysis offers a sustainable route towards the production of these important building blocks. Particularly promising are transaminases that catalyse the transfer of an amino group from an amine donor to a ketone or aldehyde with excellent stereoselectivity. For example, an engineered transaminase variant is successfully applied in the industrial synthesis of the antidiabetic drug Sitagliptin. Yet, this enzyme class is often limited with respect to activity and stability. Therefore, the identification of robust and active transaminases with extensive substrate scopes is of high importance in order to extend their application in industry and academia.

Now, Helen C. Hailes, John M. Ward and co-workers explore a domestic household for the identification of transaminases from bacteria that are adapted to extreme environments. The waste pipe of the domestic shower constitutes an ecological niche with periods of high temperatures, chemicals and surfactants. A metagenomics analysis of an environmental sample from this waste pipe led to the identification of 36 transaminases, of which 29 could be successfully expressed in E. coli for further characterization. The characterized enzymes showed high robustness, being operational in several solvents and active over a broad temperature range. For example, one enzyme was active within $\mathrm{pH}=6-11$, $20-60{ }^{\circ} \mathrm{C}, 50 \%$ DMSO and in the presence of 1-50 equivalents of the amine donor isopropylamine. Remarkably, the enzymes also showed a good substrate scope, allowing the transformation of aliphatic and cyclic ketones including heterocycles and pharmaceutically relevant motifs.

In a nutshell, the approach led to the identification of transaminases that readily display desired properties that are not usually seen in natural non-engineered enzymes. The extreme environment provided in the waste pipe of the household apparently provided an effective pressure for the evolution of more robust transaminases, which allowed these organisms to survive and grow under these conditions. This work demonstrates the value of using metagenomics for biocatalyst discovery from niche environments, and the identified enzymes constitute promising starting points for further enzyme engineering towards desired targets and reaction conditions.

\section{Jan-Stefan Völler}

Published online: 12 December 2018 https://doi.org/10.1038/s41929-018-0210-6 